\title{
Alcohol Outlets, Neighborhood Characteristics, and Intimate Partner Violence: Ecological Analysis of a California City
}

\author{
Carol B. Cunradi, Christina Mair, William Ponicki, \\ and Lillian Remer
}

\begin{abstract}
Neighborhood indicators of social disadvantage, such as poverty and unemployment, are associated with intimate partner violence (IPV). Despite the wellestablished link between heavy drinking and IPV, few studies have analyzed the contribution of alcohol outlet density to the occurrence of IPV. Greater numbers of alcohol outlets in a community may be a sign of loosened normative constraints against violence, promote problem drinking among at-risk couples, and provide environments where groups of persons at risk for IPV may form and mutually reinforce IPV-related attitudes, norms, and problem behaviors. This study used ecological data to determine if alcohol outlet density number of bars, restaurants serving alcohol, and off-premise outlets per unit area) is related to rates of IPV-related police calls and IPV-related crime reports in Sacramento, California. Separate analyses for IPV calls and crime reports were conducted using Bayesian space-time models adjusted for area characteristics (poverty rate, unemployment rate, racial/ethnic composition). The results showed that each additional off-premise alcohol outlet is associated with an approximate 4\% increase in IPV-related police calls and an approximate 3\% increase in IPV-related crime reports. Bars and restaurants were not associated with either outcome. The findings suggest that alcohol outlet density, especially off-premise outlets, appear to be related to IPV events. Further research is needed to understand the mechanisms by which neighborhood factors, such as alcohol outlet density, affect IPV behaviors. Understanding these mechanisms is of public health importance for developing environmental IPV prevention strategies, such as changes in zoning, community action, education, and enforcement activities.
\end{abstract}

KEYWORDS Intimate partner violence, Neighborhoods, Alcohol outlet density, Police data

\section{INTRODUCTION}

Aspects of the urban environment appear linked to the occurrence of intimate partner violence (IPV). For example, census-based measures of neighborhood social disadvantage, such as percentage of households living below the Federal poverty line and area-wide unemployment level, are associated with IPV risk, even after

Cunradi, Mair, Ponicki, and Remer are with the Prevention Research Center, Pacific Institute for Research and Evaluation, Berkeley, CA, United States; Mair is with the School of Public Health, University of California, Berkeley, CA, United States.

Correspondence: Carol B. Cunradi, Prevention Research Center, Pacific Institute for Research and Evaluation, Berkeley, CA, United States. (E-mail: Cunradi@prev.org) 
accounting for individual-level socioeconomic factors. ${ }^{1-4}$ Self-reported neighborhood social disorder (e.g., respondent report of crime, drug selling, street fights, empty or abandoned buildings, graffiti) was an independent risk factor for IPV among a national sample of married/cohabiting men and was shown to moderate the association between drinking and IPV among the sample's married/cohabiting women. ${ }^{5}$ One aspect of the neighborhood environment that may be related to IPV risk but has largely gone unanalyzed is alcohol outlet density. Outlet density (i.e., geographic distribution of bars/pubs, restaurants, and off-premise outlets) may affect the likelihood of IPV through several potential mechanisms. Greater numbers of alcohol outlets within a neighborhood may: (1) indicate loosened normative constraints against violence; (2) promote problem alcohol use among at-risk couples; and (3) provide environments where groups of persons at risk for IPV may form and mutually reinforce IPV-related attitudes, norms, and problem behaviors. ${ }^{6}$ Both high neighborhood median income and high income inequality appear to be associated with greater likelihood of drinking, ${ }^{7}$ while heavy drinking may be associated with neighborhood disadvantage. ${ }^{8}$ Given this, together with the fact that heavy or problem drinking is linked to the occurrence of IPV,, 10 understanding the role of alcohol outlet density, especially in the context of socially disadvantaged neighborhoods, may hold important implications for environmental IPV prevention strategies. ${ }^{6}$

Alcohol outlet density has been found to be associated with violent assault in cross-sectional and longitudinal studies. For example, Lipton and Gruenewald ${ }^{11}$ found that bar density in California was associated with greater rates of assault, while restaurant density was associated with less violence. Alcohol outlet density significantly contributed to rates of assaultive violence in an analysis of inner-city census tracts in Kansas City, Missouri. ${ }^{12}$ Similar findings were reported in an ecologic study of outlet density and violent crime, controlling for neighborhood sociostructural factors, in Austin and San Antonio, Texas. ${ }^{13}$ In a longitudinal analysis on alcohol availability and assault, Gruenewald and Remer ${ }^{14}$ found that bars and off-premise outlets were related to assault rates over time, based on an ecological analysis of 581 California zip code areas. Yu et al. ${ }^{15}$ found that civic unrest in L.A. during 1992 led to damaged alcohol outlets and decreased alcohol sales. Beginning 1 year later, census tracts that experienced alcohol outlet closures had a greater decrease in assault rates.

To date, few studies have analyzed the association between alcohol outlet density and IPV. An ecological analysis of New Jersey municipalities by Gorman et al. ${ }^{16}$ found that a census-based composite measure of social disadvantage was strongly correlated with IPV rates, while alcohol outlet density was not. A multilevel analysis conducted by McKinney et al., ${ }^{17}$ based on a national sample of married/ cohabiting couples, found that alcohol outlet density increased the risk for male-tofemale IPV and that this relationship differed for couples who did and did not report alcohol-related problems. The purpose of the current study is to investigate the ecological association of alcohol outlet density (bars, restaurants, and off-premise outlets) with IPV-related police calls (from 2006 to 2009) and IPV-related crime reports (from 2001 to 2009) for the city of Sacramento, California while accounting for census-based indicators of poverty, unemployment, and racial/ethnic composition. Situated in the Central Valley, Sacramento is the capital and 7th largest city in the state, with a 2008 estimated population of 463,794. We hypothesized that greater numbers of outlets, especially bars and off-premise outlets, would be positively related to both IPV-related outcomes. 


\section{METHODS}

\section{Data Acquisition}

IPV-Related Police Calls. Information on IPV-related calls was collected from Sacramento Police Department dispatch data. Data was available from 2006 to 2009. Two primary codes were included in the definition of domestic violence calls. The first code (273.5) includes domestic violence calls that involved reports of physical violence between a married or dating couple. There are approximately 7,000 calls reported each year with this code. The second code (415DV) covers calls in which verbal altercation between a married or dating couple was reported. There are approximately 6,000 calls per year reported under this code. Multiple calls may have been received for the same incident (and coded differently, depending on the information that the dispatcher received). Data were available for 576 police-defined polygons called electronic data processing (EDP) grids. These are small, stable, welldefined geographic units averaging 0.17 square miles in size. The call analyses below covered 576 EDP grids across 4 years for a total of 2,304 space-time units.

IPV-Related Crime Reports. Crime report records were also obtained from the Sacramento Police Department. These records are collected and coded using FBI Uniform Crime Report (UCR) protocols. UCR data are used to measure changes in crime rates across the United States, as well as for local law enforcement administration, operation, and management. Typically, these data are generated by police officers inputting the report into the computer system immediately after an incident has been resolved (warning, citation, arrest, etc.). If a police officer responds to a call for service but no reportable crime occurred, a crime report is not generated. For this analysis, domestic violence crime reports were identified using 3 codes: the beating of a spouse or cohabitant (code 273.5), battery of a noncohabiting spouse (code 243(E)1), and the violation of a court restraining order (code 273.6(A)). Crime report counts were aggregated across 576 EDP grids for 9 years $(n=5,184$ space-time units).

Alcohol Outlets Density. Active alcohol retail license data were collected annually from the California Department of Alcohol Beverage Control. Three types of alcohol retail establishments (off-premise establishments, restaurants, and bars) were coded using license type information. Premise addresses were geocoded in ArcView 9.3.1. ${ }^{18}$ The models in this analysis used a density measure of the number of outlets of each type within a half mile of each EDP grid centroid. The 3 types of outlets were kept separate in analyses because the direction and strength of associations were expected to differ between restaurants, which often signify upscale areas within a city, and offpremise outlets, which provide less expensive alcohol and tend to be denser in less affluent neighborhoods.

Census-Based Data. Yearly estimates of US census-based characteristics were assembled by GeoLytics, Inc. ${ }^{19}$ at the census block group level. These variables included population count, percentage under $150 \%$ of poverty level, percentage high school graduates, unemployment rate, percentage Hispanic, and percentage black. The demographic and economic characteristics from the 330 Sacramento block groups were apportioned to the 576 EDP grids based on the assumption that each block group is demographically homogenous across its geographic area. This 
apportionment was performed based on the union of polygon overlays in ArcGIS 9.3.1.

\section{Data Analysis}

Each outcome measure (IPV-related calls and IPV-related crime reports) was analyzed separately using a Bayesian space-time model similar to that of Bernardinelli et al., ${ }^{20}$ implemented in WinBUGS 1.4.3 software $^{21}$ as specified in Lawson et al. 22

$$
\begin{aligned}
& \operatorname{ProbCnt}_{i, k} \sim \operatorname{Poisson}\left(\mu_{i, k}\right) \\
& \log \left(\mu_{i, k}\right)=\log \left(\operatorname{eProbCnt}_{i, k}\right)+\alpha+\xi \cdot z_{i}+\beta \cdot x_{i, k}+u_{i}+\delta_{i} \cdot t_{k}
\end{aligned}
$$

where ProbCnt ${ }_{i, k}$ refers to the Poisson probability distribution assumed to represent counts of crime reports or calls in EDP grid $i$ in year $k$. Crime report and call outcomes are regressed on exogenous measures represented by fixed and random effects. Variable eProbCnt ${ }_{i, k}$ represents the expected number of events in each grid and year if total citywide problems are distributed over space and time strictly in proportion to population. Fixed-effect parameter $\alpha$ represents an overall intercept. Temporal variation in citywide IPV risks, $\xi$, was modeled using dummy variables $z_{i}$ for each year after the first year of collected data (2007-2009 for calls, 2002-2009 for crime reports). Parameter vector $\beta$ measures the effects of exogenous zip code by year variables such as outlet densities, demographic, and economic variables.

The random effect $u_{i}$ allows for the possibility that some EDP grids have a consistently higher (or lower) than average IPV risk throughout the study period that is not explained by their values of exogenous variables. The random effect $\delta_{i}$ allows for the possibility that individual grid areas may have problem risks that are linearly increasing or decreasing over time relative to the city as a whole. Estimates of both $u_{i}$ and $\delta_{i}$ are implemented using conditional autoregressive (CAR) Bayesian priors to control for the possibility of spatial autocorrelation. This helps smooth spatial patterns by having the predictions for each EDP grid borrow strength from nearby grids' data. ${ }^{23}$

The Bayesian space-time models were allowed to burn-in for 50,000 Markov Chain Monte Carlo (MCMC) iterations, after which parameter estimates from the posterior distribution were sampled for 20,000 iterations. Models were estimated using 2 Markov chains, assuming different initial values to assure that both chains produced similar results. Preliminary analyses with both spatially autocorrelated and non-spatial random intercepts did not converge well, so the final model includes only the spatial random effects $\left(u_{i}\right)$. Traces of MCMC iterations (not shown) demonstrated good convergence for all parameters in these analyses.

The Deviance Information Criterion (DIC) was used to assess model fit. The DIC captures information about both the goodness of fit and the complexity of a model. The models presented below had the best fit according to the DIC (models with smaller DIC are better supported by the data). These models included the density of each alcohol outlet type (number of bars, restaurants, and off-premise licenses within a half-mile radius), percentage black, percentage Hispanic, and percentage under $150 \%$ of poverty line and unemployment rate. Spatial autocorrelation of both the random intercepts and growth rates were calculated using the Moran's $I$ statistic $^{24}$ in ArcGIS. This statistic can range from -1 (indicating 
dispersion) to 1 (indicating clustering), with 0 corresponding to a completely random spatial pattern.

\section{RESULTS}

Table 1 presents descriptive statistics for EDP grids. Means, standard deviations, and ranges were calculated for both the crime report data (which span the years 2001-2009) and calls data (years 2006-2009). EDP grids had an average estimated population of 826 from 2006 to 2009 and 797 from 2001 to 2009 (Table 1). An average EPD grid had approximately 1 bar, 3 off-premise outlets, and 6 restaurants within one-half mile of its center. These distributions are all skewed, with a single EDP grid having as many as 87 restaurants in a given year, for example. There were an average of 23.6 IPV-related calls per grid, with a single EDP grid reporting as many as 237 in a single year. As expected, there were fewer IPV-related crime reports than calls per grid polygon per year (mean 4.5, range 0-75).

Table 2 shows the results from the Bayesian space-time models for IPV-related calls and crime reports. The models presented here demonstrated the best model fit according to the DIC criterion. An increase of one off-premise alcohol outlet within a half mile of an EDP grid centroid was associated with an increase in the likelihood of both IPV-related calls and crime reports throughout the $95 \%$ credible interval. This suggests that each additional off-premise alcohol outlet is associated with an approximate $4 \%$ increase in IPV-related police calls and an approximate 3\% increase in crime reports. Neither bars nor restaurants had a clear association with IPV calls or crime reports. Several census-based characteristics were associated with

\section{TABLE 1 Descriptive statistics}

\begin{tabular}{|c|c|c|c|c|}
\hline & Mean & SD & Minimum & Maximum \\
\hline \multicolumn{5}{|c|}{ IPV-related calls (2006-2009; $n=2,304$ EDP grids) } \\
\hline Population $^{\mathrm{a}}$ & 826.1 & 781.0 & 1.7 & 5717.3 \\
\hline No. of bars ${ }^{b}$ & 0.9 & 2.3 & 0 & 18 \\
\hline No. of off-premise outlets ${ }^{b}$ & 3.2 & 3.5 & 0 & 17 \\
\hline No. of restaurants ${ }^{b}$ & 6.1 & 11.9 & 0 & 87 \\
\hline Percentage of Black ${ }^{a}$ & 13.3 & 10.2 & 0 & 56.4 \\
\hline Percentage of Hispanic ${ }^{a}$ & 24.1 & 11.8 & 0.4 & 65.9 \\
\hline Percentage of below $150 \%$ poverty line ${ }^{a}$ & 29.4 & 18.7 & 0.9 & 83.2 \\
\hline Percentage of unemployed ${ }^{a}$ & 11.0 & 12.7 & 0 & 97.9 \\
\hline No. of IPV-related calls & 23.6 & 28.8 & 0 & 237 \\
\hline \multicolumn{5}{|c|}{ IPV-related crime reports (2001-2009; $n=5,184$ EDP grids) } \\
\hline Population $^{\mathrm{a}}$ & 797.0 & 736.6 & 1.6 & 5717.3 \\
\hline No. of bars ${ }^{b}$ & 1.0 & 2.3 & 0 & 19 \\
\hline No. of off-premise outlets ${ }^{b}$ & 3.2 & 3.5 & 0 & 17 \\
\hline No. of restaurants ${ }^{b}$ & 5.7 & 11.1 & 0 & 87 \\
\hline Percentage of Black ${ }^{a}$ & 13.3 & 10.3 & 0 & 58.5 \\
\hline Percentage of Hispanic ${ }^{a}$ & 22.9 & 11.5 & 0.3 & 65.6 \\
\hline Percentage of below $150 \%$ poverty line ${ }^{a}$ & 29.9 & 19.1 & 0.9 & 86.4 \\
\hline Percentage of unemployed ${ }^{a}$ & 9.5 & 11.0 & 0. & 97.9 \\
\hline No. of IPV-related crime reports & 4.5 & 6.1 & 0 & 75 \\
\hline
\end{tabular}

${ }^{a}$ Estimates derived from proportioning census blocks to EDP grids based on the union of polygon overlays

${ }^{b}$ Number of outlets calculated as the number within 1/2 mile of EDP grid centroid 


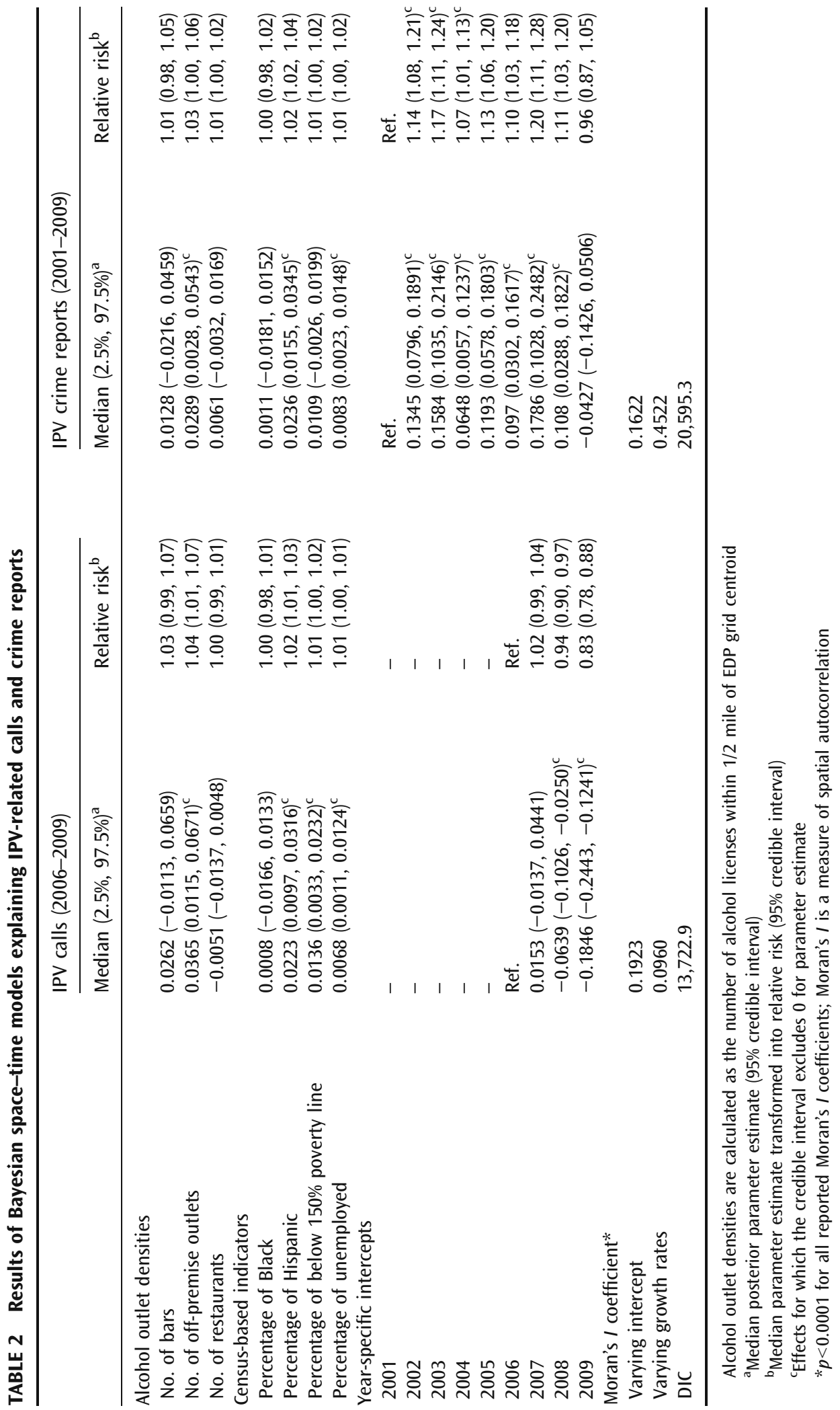


IPV-related crime reports and calls. Higher percentages of both Hispanic and unemployed residents were associated with increases in calls and crime reports, while a greater percent of residents below $150 \%$ poverty line was associated with increased risk of IPV calls. The likelihood of IPV-related calls and crime reports varied across years (Table 2). For IPV-related crime reports, 2001 and 2009 had the lowest risk + and 2007 the highest. The likelihood of IPV-related calls decreased from 2006 to 2009. The Moran's I coefficients for both the varying intercepts and growth rates indicate very significant positive spatial autocorrelation $(p<0.0001)$.

\section{DISCUSSION}

Consistent with the hypotheses of this study, the results suggest that greater densities of off-premise alcohol outlets are associated with increased risk for both policereported IPV calls and crime reports in the city of Sacramento. While the geography of this study is limited to one urban area, and the findings need to be replicated in other geographic locales, study results are in general accord with a considerable body of research linking alcohol outlet density with numerous problem outcomes, including violent crime, ${ }^{11,13,25}$ child maltreatment, ${ }^{26}$ alcohol-related car crashes, ${ }^{27}$ suicide, ${ }^{28}$ and underage and young adult injuries. ${ }^{29}$ Although the median parameter estimates reported in Table 2 are consistent with the hypothesis that IPV rates are positively related to bar densities, the credible intervals suggest that this finding is not compelling in either analysis. This is in contrast with the findings of McKinney et al., ${ }^{17}$ who reported that on-premise alcohol outlet density (bars and restaurants), but not off-premise outlet density, was significantly associated with increased risk of male-to-female violence among a national sample of married/cohabiting couples. Moreover, McKinney and colleagues ${ }^{17}$ found that neither type of alcohol outlet density was associated with risk for female-to-male partner violence among the sample's couples. Significant methodological differences between the 2 studies (e.g., longitudinal vs. cross-sectional data; single urban area vs. national sample; ecological vs. multilevel analysis) may partially account for the dissimilarities in findings. Clearly, further research is needed to determine if (and how) alcohol outlet density is differentially related to risk for common couple violence ${ }^{30}$ as well as more severe manifestations of IPV that result in police calls or crime reports. ${ }^{31}$

To our knowledge, this study is the first to make use of a Bayesian spatial modeling approach to analyze alcohol outlet density in relation to IPV-related outcomes. These models include random effects allowing for the possibility that individual grid areas (a unit of geography averaging half a block group) have levels or growth rates of IPV indicators that are consistently higher or lower than for the city of Sacramento as a whole. The modeling strategy makes use of CAR priors to explicitly control for spatial autocorrelation, the tendency of IPV-related rates between neighboring grid areas to be correlated with one another. Spatial autocorrelation among geographic units violates the standard regression assumption of unit independence, which can lead to biased results from methods that do not take account of this issue. ${ }^{23}$ The highly-significant spatial autocorrelations (Moran I statistics) for the random intercepts and growth rates reported in Table 2 provide strong support for this modeling approach. The consistency of results between the 2 sets of analyses reported above is reassuring given the differing time periods analyzed (2006-2009 for IPV calls, 2001-2009 for crime reports) and the differences in characteristics between calls and crime reports (the data suggest roughly 5 IPV calls for each crime report, which may reflect differences in case 
severity and other factors). The similar findings for calls and crime reports suggest that the results are driven by differences in underlying IPV rates rather than idiosyncrasies of the outcome measures.

There are strengths and limitations associated with using police-reported IPV calls and crime report data in the present study. Regarding strengths, police data represent an official or objective measure of IPV events within a bounded time frame and metropolitan locale. As such, they are a useful resource for public health surveillance of IPV. ${ }^{32}$ In terms of limitations, several points should be noted. First, the public crime report records used in this study do not include any personal characteristics about the victim and/or perpetrator for each IPV event (e.g., gender, age, level of violence, injury, etc.) nor whether the incident resulted in an arrest. Second, the dispatch records do not allow us to determine whether each IPV police call represented a unique event, or whether multiple calls were received per event.

Moreover, a key limitation of the study is that ecological data do not allow us to determine what underlying mechanisms explain the association between off-premise alcohol outlets and both IPV-related outcomes. For example, we cannot infer whether density of off-premise outlets increases frequency of drinking, and thereafter IPV, or whether the presence of alcohol outlets relates to other (unmeasured) structural or social processes. Multilevel analytic models that include individual and couple survey data, along with ecological information about the neighborhood environments in which couples reside, are needed to test how environmental factors, such as alcohol outlet density, increase couple risk for IPV. Understanding the mechanisms that link neighborhood environmental factors to IPV is of public health importance for developing environmental IPV prevention strategies, such as changes in zoning, community action, education, and enforcement activities. ${ }^{6}$

In conclusion, this ecological study makes an important contribution to research on neighborhood environmental factors and IPV. The findings indicate that offpremise alcohol outlet density is associated with increases in IPV-related police calls and crime reports even after accounting for neighborhood racial/ethnic composition, poverty, and unemployment within 576 small, police-defined geographic areas in Sacramento. Further research is needed to investigate mechanisms through which alcohol outlet density may increase risk for IPV in this and other urban areas.

\section{ACKNOWLEDGMENTS}

The authors would like to thank the Sacramento Police Department for providing data on IPV-related police calls and crime reports and for technical assistance. We also gratefully acknowledge Dr. Paul Gruenewald for his support and guidance on the use of Bayesian space-time modeling techniques. The project described was supported by grant number 1 R01AA017705-02 from the National Institute on Alcohol Abuse and Alcoholism; Carol Cunradi, Principal Investigator. The content is solely the responsibility of the authors and does not necessarily represent the National Institute on Alcohol Abuse and Alcoholism or the National Institutes of Health.

OPEN ACCESS This article is distributed under the terms of the Creative Commons Attribution Noncommercial License, which permits any noncommercial use, distribution, and reproduction in any medium, provided the original author(s) and source are credited. 


\section{REFERENCES}

1. O’Campo P, Gielen A, Faden R, Xue X, Kass N, Wang M-C. Violence by male partners against women during the childbearing year: a contextual analysis. Am J Public Health. 1995; 85(8): 1092-7.

2. Cunradi CB, Caetano R, Clark C, Schafer J. Neighborhood poverty as a predictor of intimate partner violence among white, black, and Hispanic couples in the United States: a multilevel analysis. Ann Epidemiol. 2000; 10(5): 297-308.

3. Cunradi CB, Caetano R, Schafer J. Alcohol-related problems, drug use, and male intimate partner violence severity among US couples. Alcohol Clin Exp Res. 2002; 26(4): 493-500.

4. Fox GL, Benson ML. Household and neighborhood contexts of intimate partner violence. Public Health Rep. 2006; 121: 419-27.

5. Cunradi CB. Drinking level, neighborhood social disorder, and mutual intimate partner violence. Alcohol Clin Exp Res. 2007; 31(6): 1012-9.

6. Cunradi CB. Neighborhoods, alcohol outlets and intimate partner violence: addressing research gaps in exploratory mechanisms. Int J Environ Res Public Health. 2010; 7: 799-813.

7. Galea S, Ahern J, Tracy M, Vlahov D. Neighborhood income and income distribution and the use of cigarettes, alcohol, and marijuana. Am J Prev Med. 2007; 32(6 Suppl): S195-202.

8. LaVeist TA, Wallace JM Jr. Health risk and inequitable distribution of liquor stores in African American neighborhoods. Soc Sci Med. 2000; 5: 613-7.

9. Leonard KE. Alcohol and intimate partner violence: when can we say that heavy drinking is a contributing cause of violence? Addiction. 2005; 100: 422-5.

10. Klostermann KC, Fals-Stewart W. Intimate partner violence and alcohol use: exploring the role of drinking in partner violence and its implications for intervention. Aggression Violent Behavior. 2006; 11: 587-97.

11. Lipton R, Gruenewald PJ. The spatial dynamics of violence and alcohol outlets. J Stud Alcohol. 2002; 63(2): 187-95.

12. Reid RJ, Hughey J, Peterson NA. Generalizing the alcohol outlet-assaultive violence link: evidence from a U.S. midwestern city. Subst Use Misuse. 2003; 38(14): 1971-82.

13. Zhu L, Gorman DM, Horel S. Alcohol outlet density and violence: a geospatial analysis. Alcohol Alcohol. 2004; 39: 369-75.

14. Gruenewald PJ, Remer L. Changes in outlet densities affect violence rates. Alcohol Clin Exp Res. 2006; 30(7): 1184-93.

15. Yu Q, Scribner R, Carlin B, et al. Multilevel spatio-temporal dual changepoint models for relating alcohol outlet destruction and changes in neighbourhood rates of assaultive violence. Geospat Health. 2008; 2(2): 161-72.

16. Gorman DM, Labouvie EW, Speer PW, Subaiya AP. Alcohol availability and domestic violence. Am J Drug Alcohol Abuse. 1998; 24(4): 661-73.

17. McKinney CM, Caetano R, Harris TR, Ebama MS. Alcohol availability and intimate partner violence among US couples. Alcohol Clin Exp Res. 2009; 33(1): 169-76.

18. ArcGIS9 ArcView. [computer program]. Version 9.3.1. Redlands, CA: ESRI (Environmental Systems Research Institute Inc.); 2009.

19. Estimates 2001-2008 Premium [computer program]. Version 1.0. Newark, NJ: GeoLytics Inc.; 2009.

20. Bernardinelli L, Clayton D, Pascutto C, Montomoli C, Ghislandi M, Songini M. Bayesian analysis of space-time variation in disease risk. Stat Med. 1995; 14(21-22): 2433-43.

21. Lunn DJ, Thomas A, Best N, Spiegelhalter D. WinBUGS-a Bayesian modelling framework: concepts, structure, and extensibility. Stat Comput. 2000; 10(4): 325-37.

22. Lawson AB, Browne WJ, Vidal Rodeiro CL. Disease mapping with WinBUGS and MLwiN. Chichester: Wiley; 2003.

23. Waller LA, Gotway CA. Applied spatial statistics for public health data. Hoboken: Wiley; 2004. 
24. Moran PAP. Notes on continuous stochastic phenomena. Biometrika. 1950; 37: 17-33.

25. Gruenewald PJ, Freisthler B, Remer L, LaScala EA, Treno A. Ecological models of alcohol outlets and violent assaults: crime potentials and geospatial analysis. Addiction. 2006; 101: 666-77.

26. Freisthler B, Midanik LT, Gruenewald PJ. Alcohol outlets and child physical abuse and neglect: applying routine activities theory to the study of child maltreatment. J Stud Alcohol. 2004; 65: 586-92.

27. Treno AJ, Johnson FW, Remer L, Gruenewald PJ. The impact of outlet densities on alcohol-related crashes: a spatial panel approach. Accid Anal Prev. 2007; 39: 894-901.

28. Johnson FW, Gruenewald PJ, Remer LG. Suicide and alcohol: do outlets play a role? Alcohol Clin Exp Res. 2009; 33(12): 2124-33.

29. Gruenewald PJ, Freisthler B, Remer L, LaScala EA, Treno AJ, Ponicki WR. Ecological associations of alcohol outlets with underage and young adult injuries. Alcohol Clin Exp Res. 2010; 34(3): 519-27.

30. Johnson MP. Patriarchal terrorism and common couple violence: two forms of violence against women. J Marriage Fam. 1995; 57: 283-94.

31. Bachman R, Coker AL. Police involvement in domestic violence: interactive effects of victim injury, offender's history of violence and race. Violence Vict. 1995; 10: 91-106.

32. Joshi M, Sorenson SB. Intimate partner violence at the scene: incident characteristics and implications for public health surveillance. Eval Rev. 2010; 34(2): 116-36. 